\title{
Quantifying Causal Contributions in Earth Systems by Normalized Information Flow
}

\author{
Chin-Hsien Cheng ${ }^{1,2}$, Simon A. T. Redfern ${ }^{1}$ \\ ${ }^{1}$ Asian School of the Environment, Nanyang Technological University, 50 Nanyang Avenue, Singapore 639798 \\ ${ }^{2}$ Joint International Research Laboratory of Climate and Environment Change, Nanjing University of Information \\ Science and Technology (NUIST), Nanjing 210044, China
}

Correspondence to: Simon A. T. Redfern (simon.redfern@ntu.edu.sg)

\begin{abstract}
To understand the plethora of important processes that are characterized by their complexity, from global pandemics to global climate change, it may be critical to quantify causal contributions between time series variables. Here, we examine an empirical linear relationship between the rate of changing causes and effects with various multipliers. Sign corrected normalized information flow $\left(n I F_{c}\right)$ tends to provide the best estimates of causal contributions, often in situations where such causality is poorly reflected by regressions. These include: i) causal contributions with alternating feedback (correlation) sign, ii) significant causal time-lags, iii) significant noise contributions, and iv) comparison among many causes to an overall mean effect, especially with teleconnection. Estimates of methane-climate feedbacks with both observational and Earth system model CESM2 data are given as examples of nonlinear process quantification and model assessment. The relative causal contribution is hypothesized to be proportional to $|n I F|$, i.e. the ratio between entropy (degree of uncertainty) received from the cause-variable (i.e. information flow, $|I F|$ ) and the total entropy change of the effect-variable. Large entropy, associated with noise, deteriorates the estimates of total entropy change, and hence $n I F$, while the proportional relationship between the relative causal contribution and $I F$ improves.
\end{abstract}

Keywords: causality, information flow, causal contributions, methane-climate feedback, model assessment 


\section{Introduction}

Causality is one of the foundations of scientific understanding and progress. Causality, being one of the foundations of scientific understanding and progress, continues to expand its application in various research disciplines in recent years, including in biomedical science (Russo and Williamson, 2011; Rasmussen et al., 2016; Lin and Ikram, 2020; Friston et al., 2020), neuroscience (Seth et al., 2015; Chen et al., 2016; Stokes and Purdon, 2017; Hill et al., 2017; Barnett et al., 2018), artificial intelligience (Pearl, 2019; Luo et al., 2020), economics (Granger, 1969; Varian, 2016; Athey and Imbens, 2017; Andor and Fels, 2018). For Earth sciences, causation is important, for example, for detecting causal signals and testing hypothesis against observed data (Sugihara et al., 2012; Stips et al., 2016; Winkler et al., 2021), evaluating, constraining, and improving climate models (Cox et al., 2018; Bai et al., 2018; Hall et al., 2019; Verbitsky et al., 2019; Vázquez-Patiño et al., 2020; Nowack et al., 2020), and estimating attribution of extreme or local events to climate or other global change (Ornes, 2018; Pfrommer et al., 2019; Swain et al., 2020). The application of various causal methods to Earth sciences has been reviewed by Runge et al (2019), where the challenges of such methods are discussed, especially those arising from the nonlinear and spatiotemporal variation of complex processes. Runge et al (2019) also suggested a way forward for Earth sciences, by combining observational causal inference and physical modelling. Beyond the likelihood of causality, the development of methods that are capable of quantifying physical causal contributions between time series is, therefore, key to resolving and understanding causality in Earth systems science process.

The progress of causal research has been fueled by the continued development and improvement of analytical tools for assessing causal influences, from the Nobel-prize winning Granger causality developed in 1960s (Granger, 1969) to the Shannon entropy-based information transfer (flow) (Schreiber, 2000) in the $21^{\text {st }}$ century. Among various methods, the information flow (IF) (Liang, 2014, 2016, 2018) and its normalized form $(n I F)$ (Liang, 2015, 2016) derived by Liang are relatively new, yet rigorously established, measures of causality between two dynamical events realized in time series. Currently, this method has mainly been applied in Earth sciences, with examples of its application including confirmation of the contribution of anthropogenic greenhouse gases (GHGs) to global warming in the post-industrial period (Stips et al., 2016) and in the forecasting the tropical cyclone genesis (Bai et al., 2018). Nevertheless, although $I F$ and $n I F$ are good quantitative measures of causality strength, their qualitative application has been limited to tuning the final form of other statistical models, such as improving regression-based correlation with multiple potential factors by selecting only factors with significant causal influence (Bai et al., 2018). This indirect qualitative application of $I F$ or $n I F$ does not always fully utilize the determined causality, especially if we wish to quantify the varying interdependent contributions between causally related variables instead of simply filtering out those variables that are deemed to have insignificant causal influence. A direct quantitative application of $I F$ and $n I F$ for building causal models, however, requires robust verification. Here, we explore, empirically, the conditions under which $I F$ or $n I F$ may be suitable for quantifying causal contribution trends. We have done this using mock-up interdependent variables, followed by demonstrating its application with a real-world problem: methane-climate feedback, and propose a hypothesis to rationalize the justifiable use of $I F$ and $n I F$ in determining and quantifying causal contributions. 


\section{Methods}

\subsection{The Concept}

The assessment in this work is to explore if we can apply the magnitudes of $I F$ or $n I F$, i.e. measures for flows of the amount of information or degree of uncertainty, equivalent to Shannon entropy (Liang, 2014, 2015, 2016, 2018), as measures for quantifying causal contributions, since stronger information flow implies stronger causal contributions. Therefore, we make an empirical comparison between the $I F, n I F$, and regressions based on a common form correlating the interdependency of two time-series variables, say $X$ and $Y$, estimating the contribution of variable $X$ to the change of variable $Y$ (i.e. $\partial Y_{X} / \partial t$ in equation 1).

$\frac{\partial Y_{X}}{\partial t}=\alpha \times$ multiplier $\times \frac{d X}{d t}$

We express this as a partial derivative, $\partial Y_{X} / \partial t$, since it only equates to $d Y / d t$ when other non- $X$ variables are held constant (i.e. zero noise contribution), and hence it can represent the causal contribution to the effect-variable $Y$ for cases with fluctuating noise. On the other hand, the total derivative $d X / d t$ represents the actual rate of change of cause-variable $X$. The multiplier varies among methods. For linear regression, it is $m R^{2}$ where $m$ is given by $Y$ $=m X+c$ and $R$ is the correlation coefficient. For second order regression, the multiplier is $M_{2} R^{2}$ with $M_{2}=2 a X+b$, the differential of $Y=a X^{2}+b X+c$ (with subscript 2 in $M_{2}$ denoting 2nd order regression). However, correlation via such regressions does not imply causality and could lead to inaccurate conclusions. We, therefore, would like to assess if we could fit $|I F|$ or $|n I F|$ as the multiplier with its positive or negative sign corrected by the sign of correlation coefficient in the regression, corresponding to either positive or negative feedback (correlation). We denote the sign-corrected $I F$ and $n I F$ as $I F_{c}$ and $n I F_{c}$, respectively. Since both $R^{2}$ and $|n I F|$ lie in the range 0 to 1 , we also compare the estimates using $m|n I F|$ as the multiplier in equation 1. Hence, five multipliers are examined: $m R^{2}, M_{2} R^{2}, I F_{c}, n I F_{c}$, and $m|n I F|$. The parameter $\alpha$ is a calibration factor obtained by comparing the observed $d Y / d t$ or designed $\partial Y_{X} / \partial t$ with the product of multiplier and $d X / d t$. Theoretically, $\alpha=1$ for regression but we allow variability here.

$I F_{X \rightarrow Y}=\frac{C_{Y Y} C_{Y X} C_{X, d Y}-C_{Y X}^{2} C_{Y, d Y}}{C_{Y Y}^{2} C_{X X}-C_{Y Y} C_{Y X}^{2}}$

where $C_{Y X}$ is the covariance between variables $Y$ and $X$, and $C_{X, d Y}$ is the covariance between $X$ and $\dot{Y}$, given by the series approximation of $d Y / d t$ using Euler forward differencing $\left(\dot{Y}_{n}=\left(Y_{n+1}-Y_{n}\right) / \Delta t\right)$. The same system of notation applies to $C_{X X}, C_{Y Y}$, and $C_{Y, d Y}$ too.

The normalized information flow (Liang, 2015) is given by dividing the $I F$ by a normalizing factor, $Z$.

$n I F_{X \rightarrow Y}=\left|I F_{X \rightarrow Y}\right| / Z_{X \rightarrow Y}$

$Z_{X \rightarrow Y}=\left|I F_{X \rightarrow Y}\right|+\left|\frac{d H_{Y}^{*}}{d t}\right|+\left|\frac{d H_{Y}^{\text {noise }}}{d t}\right|$ 

the rate of change of $H_{Y}$ due to $Y$ itself (first term) and the contribution from noise (second term). Note that for simplicity we consider the self-dependent contribution $\left(1^{\text {st }}\right.$ term $)$ as part of the noise.

Since the original positive/negative sign of $I F$ refers to the increasing/decreasing trend of uncertainty (or decreasing/increasing trend of predictability) (Liang, 2014, 2018), to determine the direction of positive vs negative feedback, we apply a "sign-corrected" $n I F_{c}$ and $I F_{c}$ taking the sign given by the Pearson correlation coefficient between variables $X$ and $Y$ (i.e. $R_{X Y}$ ). Magnitudes indicate the strength of causality.

$I F_{c, X \rightarrow Y}=\left|\frac{C_{Y Y} C_{Y X} C_{X, d Y}-C_{Y X}^{2} C_{Y, d Y}}{C_{Y Y}^{2} C_{X X}-C_{Y Y} C_{Y X}^{2}}\right| \times\left( \pm 1\right.$, based on $\left.R_{X Y}\right)$

$n I F_{c, X \rightarrow Y}=\left|I F_{X \rightarrow Y}\right| /\left|Z_{X \rightarrow Y}\right| \times\left( \pm 1\right.$, based on $\left.R_{X Y}\right)$

By combining equations 1, 3 and 4 into equation 7, for $n I F_{c}$ as the multiplier, we are testing a hypothesis whether the relative causal contribution, $\left(\partial Y_{X} / \partial t\right) /(d X / d t)$, is proportional to $\left|n I F_{X \rightarrow Y}\right|$, the fractional entropy received from cause-variable (i.e. $I F_{X \rightarrow Y}$ ) over the total entropy change of effect-variable $Y$, in other words, whether the fractional uncertainty contributed by the cause-variable is proportional to its physically measured relative causal contribution to the effect-variable. Alternatively, for $I F_{c}$ as the multiplier, we are testing if Shannon entropy from cause/-variable alone could measure the relative causal contributions.

$\frac{\partial Y_{X} / \partial t}{d X / d t} \propto\left|n I F_{X \rightarrow Y}\right|=\frac{\left|I F_{X \rightarrow Y}\right|}{\left|I F_{X \rightarrow Y}\right|+\text { increase in marginal entropy } H_{Y}}$

\subsection{Assessing the Multipliers using 1D and 3D Mock-Up Data}

Real-world applications of the concept of information flow are likely to involve multi-dimensional datasets. We have carried out an assessment of the method firstly on a one-dimensional (1D) example and subsequently on a three-dimensional (3D) example. We explore the relationships between interdependent variables $X$ and $Y$, representing a cyclic causal interference or a feedback loop. In the 3D context we make an analogy to climate systems, which typically concern data expressed in terms of longitude (lon), latitude (lat), and time $(t)$ coordinates across the globe's surface (Fig. 1). We focus on a scenario with highly fluctuating causal contributions from $X$ to $Y$ (or vice versa) and a relatively weakly fluctuating noise contribution which can be due to factors other than $X$ or $Y$. This example is used because it is a rather common scenario, especially pertinent to studies that attempt to distinguish strongly fluctuating causal contributions from relatively stable low-noise contributions. Such studies could include, as an example, the natural feedbacks behind climate change, such as the climate-dependent natural emissions of methane $\left(\mathrm{CH}_{4}\right)$. In such case, the relative causal contribution described in equation 7 is then the instantaneous climate feedback strength expressed as concentration change per degree temperature change. These causal contributions to the concentration change, as well as the associated feedback strength, may show significant 
fluctuations on seasonal, interannual and multi-decadal periods (Cheng and Redfern, under review), while such fluctuations sit atop more stable (generally growing) background trends due to historical anthropogenic emissions.

In our empirical assessment, mock-up data were obtained from designed interdependent functions with 1D-to-1D variables (Fig. 1a, Figs. 2-4 and Figs. S1-S6), cause-maps of 3D-to-global-mean-1D variables (distribution and variability of contribution from causes, Fig. 1d, Fig. 5 and Figs. S7-9), and effect-maps of global-mean-1D-to-3D variables (distribution and variability of contribution as effects, Fig. 1e, Figs. S10-S13). The multipliers between designed variables $X$ and $Y$ are estimated over a moving time range. The estimated multiplier is then multiplied by the rate of change of cause at the middle of the time-window so that the causal contribution can be estimated according to equation 1 . For the $1 \mathrm{D}$ tests, each time range spans 100 time-units, while for the 3D tests, each window consists of only 49 time-units, as could be common for studying interannual variability of monthly data (i.e. 49 months in total, representing a centered month \pm 24 months). These designed functions are generally expressed as:

$d Y / d t=\partial Y_{X} / \partial t+\partial Y_{n} / \partial t=f(d X / d t, t)+n(Y, t)$, and $d X / d t=\partial X_{Y} / \partial t+\partial X_{o} / \partial t=g(d Y / d t, t)+o(X, t)$

where $f$ and $g$ are the interdependent contributions, designed as the sum of two trigonometric terms with varying frequencies and a linear term. The trigonometric terms mimic typical climate oscillations resulted from alternating positive and negative feedbacks, such as the famous El Niño-Southern Oscillation (ENSO) cycle (Im et al., 2015). Similarly, the $n$ and $o$ are noise (including self-dependent) functions designed to include three terms, either a constant plus two changing trigonometric terms, or a constant plus a changing trigonometric term plus a linear term (see Table S1 for the exact expressions of functions). Most of the time the noise function is positive to mimic the long-term historical net atmospheric $\mathrm{CH}_{4}$ accumulation due to the increased anthropogenic activity. For the 3D-to-1D cause-map tests (Fig. 1d), we further consider the presence (Figs. 5 and S7) or absence (Figs. S8-S9) of interdependent teleconnection, by assigning the interdependent function based on values from the opposite side of the hemisphere (e.g. interdependency between $d X / d t$ at $60^{\circ} \mathrm{N}$ and $d Y / d t$ at $60^{\circ} \mathrm{S}$, see Fig. 1c) or same grid (Fig. 1b), respectively. For assessment with effect-maps (Fig. 1e), the same sets of these interdependent variables are applied (Figs. S10-S13).

(a)

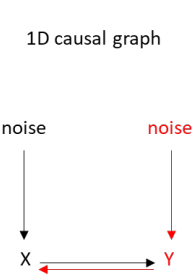

(b)
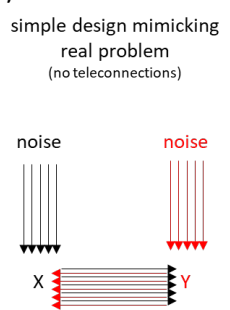

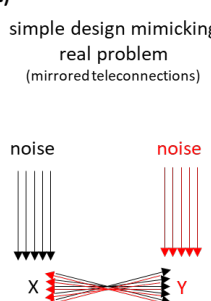

(d)

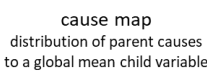
to a global mean child variable

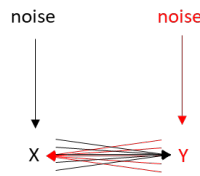
effect map
distribution of child effects from a
global mean parent variable

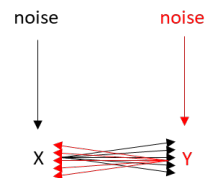

Figure 1. Illustrative causal graphs of designed $1 \mathrm{D}$ and multi-D causally interdependent variables $\mathrm{X}$ and $\mathrm{Y}$, with/without teleconnection, and the basis of estimates for cause map and effect map. 


\subsection{Example Application - Methane Climate Feedback and Model Assessment}

In addition to the mock-up data example, we also estimated the causal contribution of spatiotemporal climate factors to the variations of global atmospheric methane concentrations $\left(C_{\mathrm{CH} 4}\right)$. The climate driven contributions of $C_{\mathrm{CH} 4}$ are estimated from equation 9, which is identical to replacing $X$ in equation 1 by climate factors and $Y$ by global ocean mean $C_{\mathrm{CH} 4}$. Climate factors considered include the Land Surface Air Temperature (LSAT), Sea Surface Temperature (SST), and precipitation $(P r)$. Similar to, but more complicated than, the mock-up data cause map, these variables affect $C_{\mathrm{CH} 4}$ via both local influences on the natural methane source and sink, and remote influence via atmospheric hydroxyl radicals ( $\left.{ }^{\circ} \mathrm{OH}\right)$ or $\mathrm{CO}$ (Cheng and Redfern, under review). In addition, there are teleconnections from SST to LSAT and $\operatorname{Pr}$ before they influence $C_{\mathrm{CH} 4}$. The global and zonal mean marine surface values of $C_{\mathrm{CH} 4}$ were obtained from National Oceanic and Atmospheric Administration (NOAA) Greenhouse Gas Marine Boundary Layer Reference at https://gml.noaa.gov/ccgg/. The $0.5^{\circ} \times 0.5^{\circ} \operatorname{LSAT}, 1^{\circ} \times 1^{\circ}$ $S S T$ and $P r$ data are based on NOAA Global Historical Climatology Network (GHCN CAMS) Gridded V2, Optimum Interpolation NOAA_OI_SST_V2 and Precipitation Reconstruction over Land (PREC/L), respectively, provided by the NOAA/OAR/ESRL PSL, Boulder, Colorado, USA, from the website at https://psl.noaa.gov/. Each time window comprised 49 months (centered month \pm 24 months) as in the cause-map. Seasonal trends were removed to improve the estimates of interannual variability. The $d T / d t$ in ${ }^{\circ} \mathrm{C}_{\mathrm{yr}}{ }^{-1}$ (as well as $d P r / d t$ in mm.day ${ }^{1} \cdot \mathrm{yr}^{-1}$ and $d C_{\mathrm{CH} 4} / d t$ in ppb.yr ${ }^{-1}$ ) of each month (M) was determined as the difference between the mean temperature (or precipitation) in one year forward ( $\mathrm{M}$ to $\mathrm{M}+11$ months) and the mean in one year backward (M-12 to M-1 months). Once the 3D matrices of $n I F_{c, T} \mathbf{x} d T / d t$ or $n I F_{c, P r} \mathbf{x} d P r / d t$ were determined, the 3D data arrays were downscaled into $2 \mathrm{D}$ (lat $\mathrm{x}$ time) based on exclusive land-means to obtain the zonal mean, since ecosystem methane emissions from the oceans are suggested to be much smaller than the territorial sources (Weber et al., 2019).

$\frac{\partial C_{\mathrm{CH} 4, \text { climate }}}{\partial t}=\alpha \times$ multiplier $\times \frac{d(\text { climate factors })}{d t}$

To illustrate the application of these methods for model assessment, we have carried out similar analysis on indications of causal contribtions between simulated temperature and precipitation data obtained from the ensemble mean of Community Earth System Model 2 (CESM2) (https://esgf-node.llnl.gov/) and the same reconstructed $C_{\mathrm{CH} 4}$ observational data as described above. The model's historical end date is Dec 2014, and since we use \pm 24 months for analysis, the estimated climate-driven contributions inferred from the model data end in Dec 2012. The CESM2 is one of key models in the Coupled Model Intercomparison Project Phase 6 (CMIP6) (Danabasoglu et al., 2020). It has recently been assessed for carbon cycle $\left(\mathrm{CO}_{2}\right)$ simulation and shown reasonable capability in reflecting seasonal, but not interannual variations (Wieder et al., submitted). It is hence a good candidate to test as an example here. 


\section{Results}

\subsection{Suitable Scenarios for Applying Normalized Information Flow}

We find that for conditions where causality becomes more important, by using $n I F_{c}$ as the multiplier the causality is more clearly discerned, allowing better estimates of causality than analysis using regression. Situations where we observe this phenomenon include those when: 1) alternating positive-negative contributions (or feedbacks) especially if they act in opposition to the observed or designed general background trend (e.g. alternating contributions especially with the negative portions occurring in the context of a generally positively growing trend, Figs. 2-4, S4-S6, first column sub-Figs); 2) a significant time-lag arises between the cause and the effect (Figs. 3, S4, S6); 3) there are significant noise contributions that worsen estimates by regression more than $n I F_{c}$, in which case estimates from $I F_{c}$ are improved and may indeed become the best option when there is substantial noise (Figs. 4, S4-S6); 4) distributions of causal contributions in cause maps need to be compared, especially if there is teleconnection between causes and effects (Figs. 5 and S7 vs. Figs. S8-S9 and vs. Figs. S10-S13).
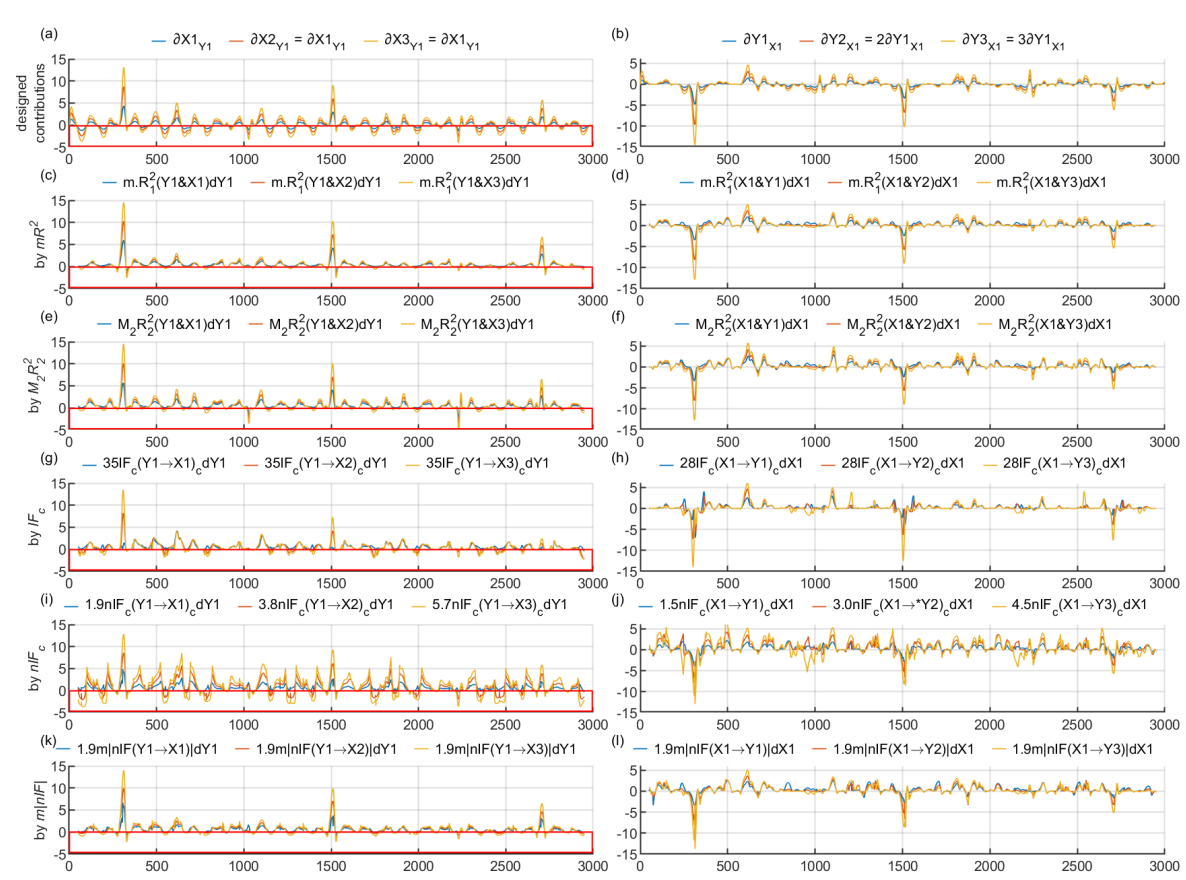

Figure 2. Assessment of methods for estimating interdependent contribution between designed 1D variables $X$ and $Y$, highlighting the conditional advantage of $n I F_{c}$ (i) for discerning the opposite (negative) contribution (red boxes) to the general (positive) trend (sub-Fig.S1a,k). The $\mathrm{x}$-axis is in time-units and the y-axis represents either the designed $(\mathbf{a}, \mathbf{b})$ or estimated contributions by various methods $\left(\mathbf{c}, \mathbf{d}\right.$ : linear regression, $\mathbf{e}, \mathbf{f}: 2^{\text {nd }}$ order regression, $\mathbf{g}, \mathbf{h}$ : information flow, $\mathbf{i}, \mathbf{j}$ : normalized information flow, $\mathbf{k}, \mathbf{l}: m|n I F|)$. Three levels of interdependency (1:2:3), reflected by blue, red, yellow, could be reflected by the calibration factor $\alpha$ for $n I F_{c}$, but not for $I F_{c}$. Refer to Fig. S1 about comparisons betweem multipliers which are estimated based on running 100 time-units of $X$ and $Y$. 

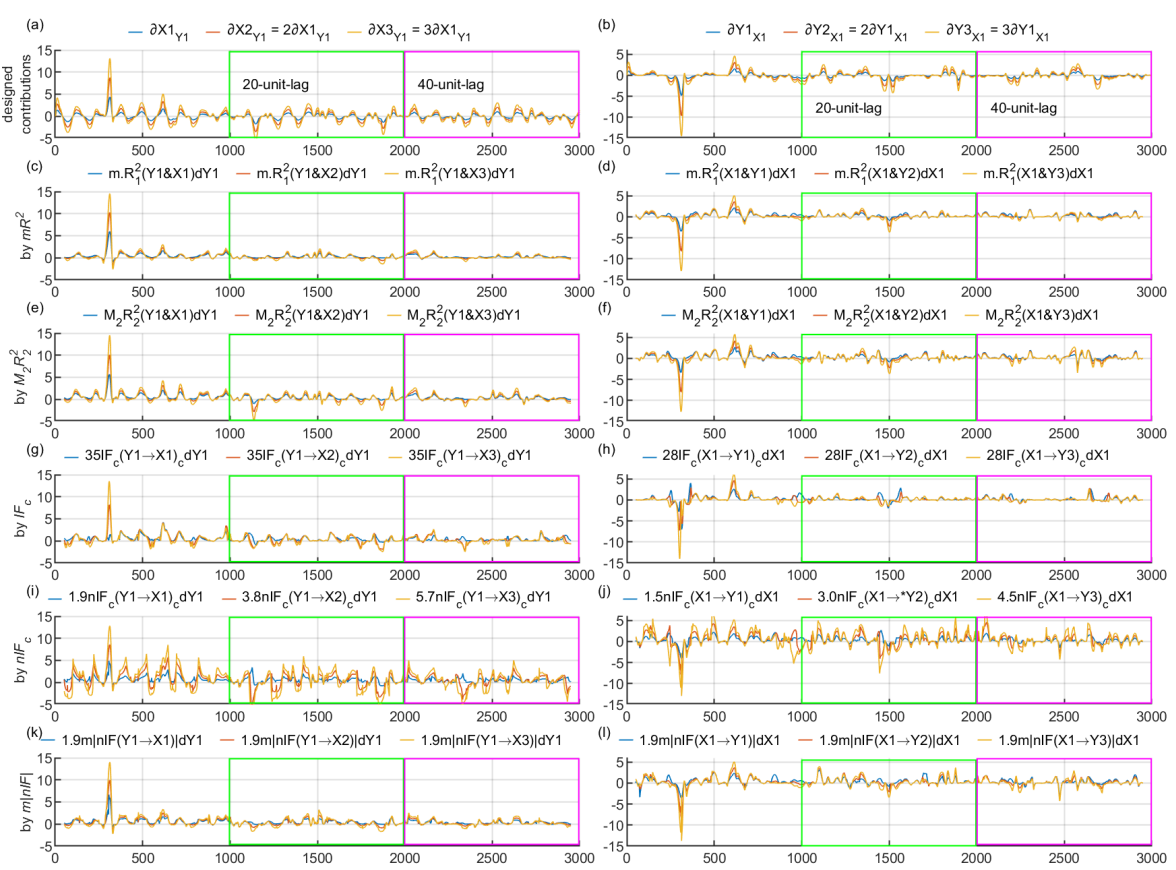

Figure 3. Assessment of methods for estimating interdependent contribution between designed 1D variables $X$ and $Y$, highlighting the conditional advantage of $n I F_{c}(\mathbf{i}, \mathbf{j})$ for significant time-lag between the cause and consequence. The green and purple boxes indicate different time-lags between the interdependency, representing $20 \%$ and $40 \%$ of the 100 -time-units in estimating the multipliers, respectively. The causal signals detected should therefore be 20 or 40 time-units ahead of the designed effects shown in (a) and (b). Figure layout as in Fig. 2. Refer to Fig. S2 for the multipliers and general trend. 

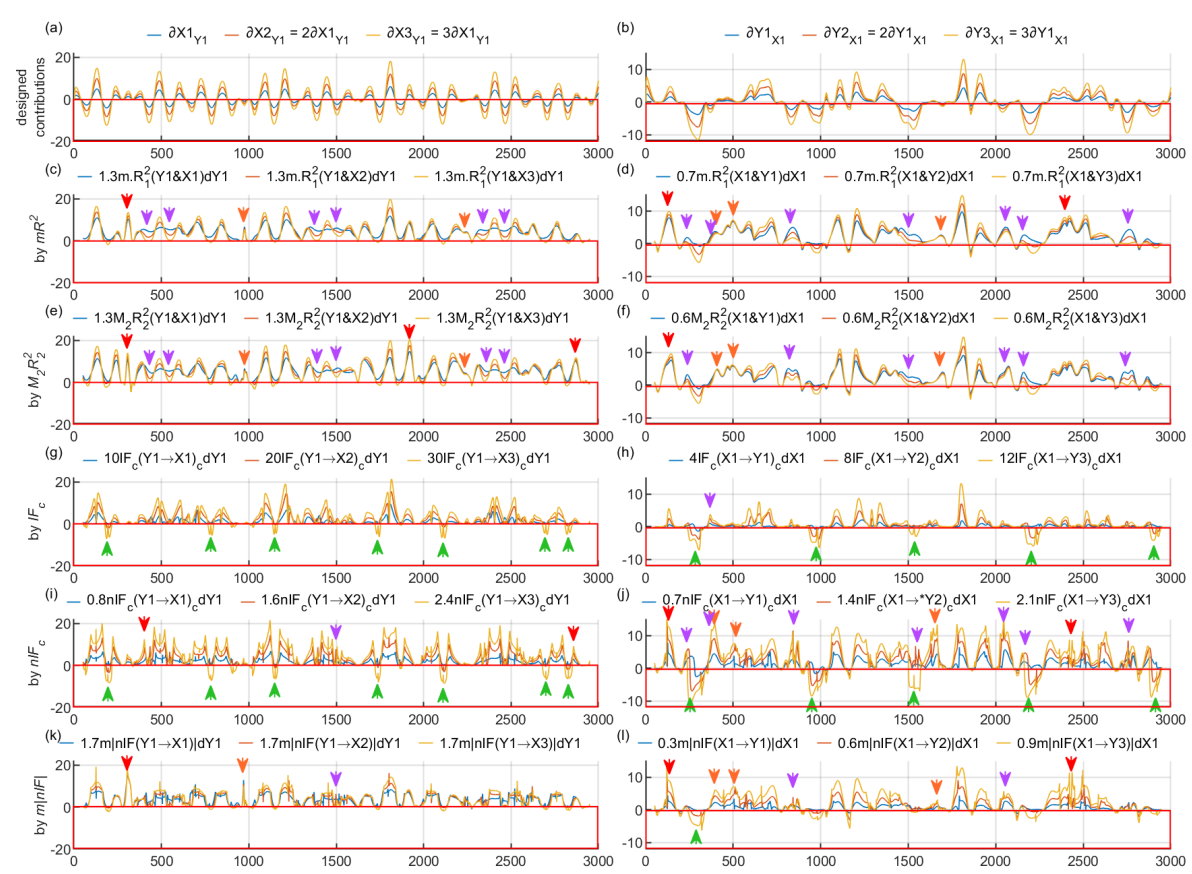

Figure 4. Assessment of methods for estimating interdependent contribution between designed 1D variables $X$ and $Y$, highlighting the impact of large noise-contributions that worsen the estimates by all methods, although that given by $I F_{c}$ appears less severly affected than the estimates obtained by regression. Figure layout as in Fig. 2 . The green arrows indicate successful capture of the opposite contribution from common trend which is more common than Fig. 2. There are instances of false signals (orange arrows), bad estimates (red arrows), and wrong feedback signs (purple arrows). It appears that $I F_{\mathrm{c}}$ becomes the best multiplier $(\mathbf{g}, \mathbf{h})$ with more consistent $|I F|$ values independent with the 1:2:3 ratio (Fig. S3), allowing practical use of constant calibration factor $\alpha$. The estimates by $I F_{\mathrm{c}}$ and $n I F_{c}$ (especially $I F_{\mathrm{c}}$ ) appear to be more resistant to incorrect feedback signs. However, if a limit is breached, the error with respect to the 1:2:3 ratio could become -1:-2:-3, while for regressions that ratio becomes similar to $-3:-2:-1$. 

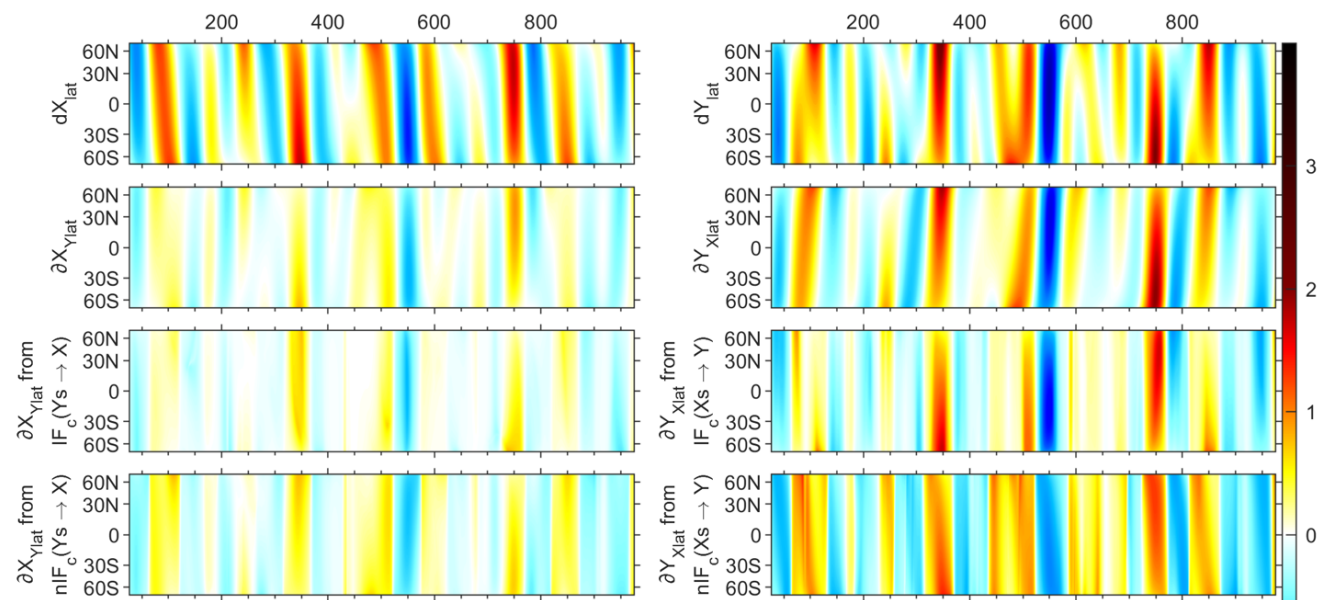

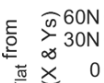

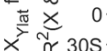

年

$\underset{\infty 30 N-}{60 N}$

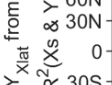

E⿱亠凶禸 $\begin{array}{r}\overline{\widehat{x}} 60 \mathrm{~N} \\ \uparrow 30 \mathrm{~N}\end{array}$

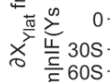

\%

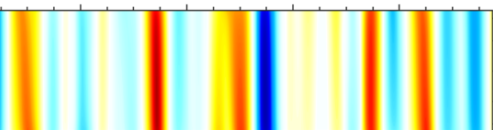

$E \underset{\infty}{\overparen{D}} 60 \mathrm{~N}$

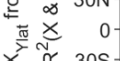

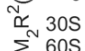

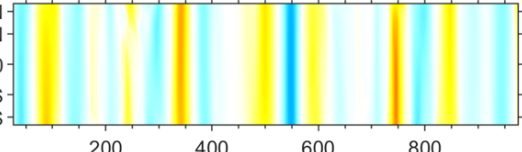

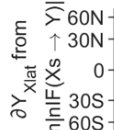

$\frac{c}{\varepsilon} 605$

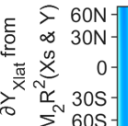

800

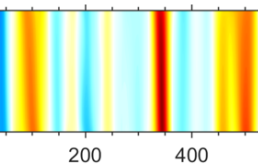

Figure 5. Assessment of methods for estimating interdependent cause-maps between designed 3D variables $X$ and $Y$, highlighting the conditional advantage of $n I F_{c}$ for detecting teleconnection. The first and second rows are the designed distributions of the rate of change of variables and interdependent contributions to that rate of change (effect-maps), respectively. The rows below are cause-map estimates by various methods, which are judged more accurate when they better represent the north-south mirror image shown in the second row. Besides $n I F_{c}$, the $I F_{c}$ with a lower interdependent contribution (left column) also provides reasonably good estimates of $\partial X_{\text {Ylat }} / d t$. 
The results from the 1D-to-1D tests are depicted in Figs. 2-4 and S1-S6. In each, the first row presents the designed values.

10 Figs. 2-4 and the first two columns in Figs. S4-6 show the interdependent contributions of $d X / d t$ from $Y$ (i.e. $\partial X_{Y} / \partial t$ ) and $d Y / d t$ from $X$ (i.e. $\partial Y_{X} / \partial t$ ). Hence, comparison of the signals between sub-Figs a-b and the estimates by various methods in rows beneath is key to this assessment. Figures S1-S3 supplement Fig.2-4 for further comparison. Sub-Figs a and b of Figs. S1-S3 show the designed $d X / d t$ and $d Y / d t$, respectively, so the differences between the first row in Figs-2-4 and Figs. S1-S3 reflect the contributions from noise signals. The middle rows in Figs. S1-S3 are key elements of the "multiplier" in the various

15 methods. These correspond to $R^{2}$ in the linear and second-order regressions, and to the absolute $|I F|$ and $|n I F|$. Each pair of interdependent $X$ and $Y$ datasets is further split into three levels of interdependency: $\partial X 1_{Y I} / \partial t$ and $\partial Y 1_{X I} / \partial t$ (blue), $\partial X 2_{Y I} / \partial t$ and $\partial Y 2_{X I} / \partial t$ (red), and $\partial X 3_{Y I} / \partial t$ and $\partial Y 3_{X I} / \partial t$ (yellow), with the latter two pairs being double and triple the values of $\partial X 1_{Y I} / \partial t$ and $\partial Y 1_{X I} / \partial t$, respectively. Comparing the results across this 1:2:3 ratio of interdependency helps indicate the feasibility of applying a constant calibration factor $\alpha$ throughout the 3000 time-units.

20 The first column of Fig. 2 highlights the alternating positive-negative contributions, with the red boxes emphasizing the negative contribution of $\partial X_{Y} / d t$ from the dominating positive $d X / d t$ (Fig. S1a) and growing $X$ (Fig. S1k). These negative signals are generally underestimated, indeed almost undetectable, in estimates by regression methods but reasonably reflected when applying estimates from $n I F_{c}$. The more frequently alternating pattern of $\partial X_{Y} / d t$ than $\partial Y_{X} / d t$ is, in fact, due to the faster phase transition in our designed trigonometric terms for the interdependent feedbacks (Table S1). If both positive and negative feedbacks occur during one timeframe of causal analysis (i.e. 100 time-units here), the opposite sign feedbacks will cancel each other and weaken the overall correlation, and the one in the original less dominant direction (i.e. the negative contributions here) may even vanish. On the other hand, the second column of Fig. 2 shows reasonable estimates given by regression methods but significant false signals are suggested by the estimate from $n I F_{c}$. Note that the calibration factor $\alpha$ for the regressions here is fixed as one, while it is adjusted for estimates by $n I F_{c}$, with the 1:2:3 ratio. This is seen in the nearly identical magnitude of

$30|n I F|$ (Fig. S1i,j) with this simple change of linear interdependency relationship. On the other hand, the magnitude of $|I F|$ (Fig. S1g,h) occasionally reflects an approximate 1:2:3 ratio when the signals are strong, however, it remains rather independent of the 1:2:3 ratio in most cases. This inconsistency makes it impractical to calibrate the interdependent contribution with a constant $\alpha$ over time-scale. The estimate by $m|n I F|$ appears to almost entirely lose its conditional advantage from $n I F_{c}$ (subFig. i vs. k), and remains less reliable than regressions in other situations (sub-Fig. d/f vs. 1). Hence, the key concerns mainly

35 lie in the conditional advantage or disadvantage that determines whether the use of regression or of $n I F_{c}$ results in better estimates.

Figure 3 focuses on an examination of the conditional advantage or disadvantage for different methods under varying time-lag of interdependency. We have imposed a three-step time lag condition. The designed contributions are set with a lag of only 1 time-unit (which is also applied to all other mock-up data shown in the other Figures) across times 2-1000, 20 time-units during 
time 1001-2000 (highlighted in green boxes), and 40 time-units during time 2001-3000 (highlighted in purple boxes). Since the estimates of the multipliers are based on 100 time-units of $X$ and $Y$ without time-lag, as we run forward from time 2-101 to $2991-3000,20 \%$ or $40 \%$ of the data in each window may contribute to potentially misleading (non causative) correlation. In the case of Fig. 3, the regression signals weaken during these two periods, but the estimates by $n I F_{c}$ remain robust without noticeable deterioration. Note that the estimated signals of causes could be around 20 or 40 time-units ahead of the designed signals of effects during these two periods.

Figure 4 shows the results of an examination of the impact of large noise contributions. The designed functions are approximately the same as those shown in Fig. 2 except the positive noise function is enlarged. This results in a more likely occurrence of opposite contribution to the common trend that could be captured by $n I F_{c}$ other than regressions, as indicated by green arrows in red boxes (comparing sub-Fig. j in Fig. 2 and 4). While the conditional advantage of $n I F_{c}$ highlighted in red boxes remains as in Fig. 2, the higher noise contributions reduce the accuracy of estimates for all methods, but the deterioration appears to be more significant when using regression. The orange, red, and purple arrows, highlight these inaccuaracies, highlighting false signals, bad estimates, and incorrect feedback signs, respectively. We note that the regression 55 method loses its capability to differentiate the 1:2:3 ratio, resulting in impractical application for a constant calibration factor. We find that incorrectly attributed feedback signs (purple arrows) are less likely for estimates by $n I F_{c}$ and $I F_{c}$ (especially $I F_{c}$ ) than using regression under this high positive noise contribution scenario. In addition, we note that the error associated with ability to reflect the 1:2:3 ratio could be as poor as -3:-2:-1 for regressions, but that ratio the worst outcome using $n I F_{c}$ and $I F_{c}$ gives a ratio of $-1:-2:-3$ once a certain limit is breached. The $n I F_{c}$-estimated peaks also appear to split into broader periods. Nevertheless, the relatively weaker interdependent contribution could help mitigate inconsistency problems when applying a constant calibration factor to $I F_{c}$ (Fig. S3g,h). This leads us to the conclusion that $I F_{c}$ appears to be the best multiplier (subFig. g,h) in these circumstances. Figures S4-S6 further show the combined conditions under medium and large noise contribution scenarios.

65 Figure 5 shows the designed and estimated contributions between two 3D variables with teleconnection operating from the opposite hemispheres (e.g. $X$ at $60 \mathrm{~N}$ are interdependent with $Y$ at $60 \mathrm{~S}$ ), projected down to two dimensions by using the zonal mean. The first row represents the designed distributions of interdependent variables $X$ and $Y$, and the second row shows the interdependent contributions (effects). The further rows below give the estimated cause-maps. The designed noise-contribution alternates between positive and negative with a rather insignificant positive bias, hence the conditional advantage in Fig. $2 \mathrm{i}$ is also insignificant. Furthermore, the time-lag is only one time-unit over the running window of time-series data, with 49 time units in each series, which limits the conditional advantage given in Fig. 3i. Nevertheless, in view of the mirrored teleconnection between north and south hemispheres, the best estimate of cause-map should also be a mirrored image of the 
$2^{\text {nd }}$ row. This mirror characteristic can be best detected in the estimates given using $I F_{c}$ and $n I F_{c}$. However, the estimates obtained by regressions and $m|n I F|$ do not provide any clear evidence of such mirroring, although the estimates by secondorder regression may manage to reflect this slightly better than those provided by first-order regression. Figure S7 gives a further example of a similar analysis, with north-south teleconnection operating at higher variability frequency, and we see the same trends. The advantage of empoying $I I F_{c}$, and $I F_{c}$ for estimating the spatial distribution in 3D-to-1D cause-maps is still apparent, but to a lower extent, in the absence of teleconnection (Fig. S8-S9). In particular, in a low noise-contribution scenario (right column maps), the estimates obtained using $n I F_{c}$, and $I F_{c}$ could become worse than those suggested from regressions. This is consistent with the findings seen in Fig. 4 and S6, suggesting that using $I F_{c}$ as the multiplier gives better results at higher noise levels. For 1D-to-3D effect-maps, regression tends to give better estimates regardless of the absence or presence of teleconnection (Fig. S10-S13). This is because there are, altogether, $360 \times 180$ time-series over the grids which contribute to a single global mean time-series in the 3D-to-1D cause-maps, emphasizing the importance of locations of cause signals in this case. This contrasts with the 1D-to-1D and 1D-to-3D effect maps where location-data of the causes are already merged into a global mean value.

\subsection{Estimated Climate-Driven Contributions to $C_{\mathrm{CH} 4}$ based on Observed and Modeled Data}

Figure 6 shows the results of estimated terrestrial climate driven contributions to the variations of $C_{\mathrm{CH} 4}$ based on the observational climatic data and the various methods discussed above. Despite the fact that only the global $C_{\mathrm{CH} 4}$ is addressed in equation 8 , the estimates given when using $n I F_{c}$ reasonably reflect the variations of reconstructed $C_{\mathrm{CH} 4}$ from observation on both temporal and zonal scales. In comparison, the estimates given by other methods are noticeably inferior. The estimates given by using $I F_{c}$ do not reflect the strong climate-driven contributions before 1992 and after 2013 so well, while the estimates given by $m R^{2}$ and $m|n I F|$ do not reflect contributions from mid-high latitudes as well as years with slower $C_{\mathrm{CH} 4}$ as clearly as does the use of $n I F_{c}$. The problem with estimates by $I F_{c}$ probably arise from its inherent, but inappropriate, assumption of a constant calibration factor $\alpha$. The underestimates by $m R^{2}$ and $m|n I F|$ likely are associated with the fluctuating causal pattern (including the change between positive and negative feedbacks, (Cheng and Redfern, under review)), especially for years with weaker climate-driven contributions. This trend is aligned with the phenomenon shown in left column of Fig. 2. The lag between cause to effect, as in Fig. 3, could also result in poor estimates when using regression methods, since the cause-signals by $n I F_{c}$ tend to lead the actual $C_{\mathrm{CH} 4}$ by months to a year or more, especially at mid-high latitudes. For $n I F_{c}$, the estimated

100 climate-driven contributions in the Northern Hemisphere $(\mathrm{NH})$ better reflect its actual zonal $C_{\mathrm{CH} 4}$ patterns as compared to signals from the Southern Hemisphere (SH). This could be due to the much higher terrestrial area in the NH and its stronger causal contribution to the global $C_{\mathrm{CH} 4}$. 
https://doi.org/10.5194/gmd-2021-196

Preprint. Discussion started: 19 July 2021

(c) Author(s) 2021. CC BY 4.0 License.

(c) (1)

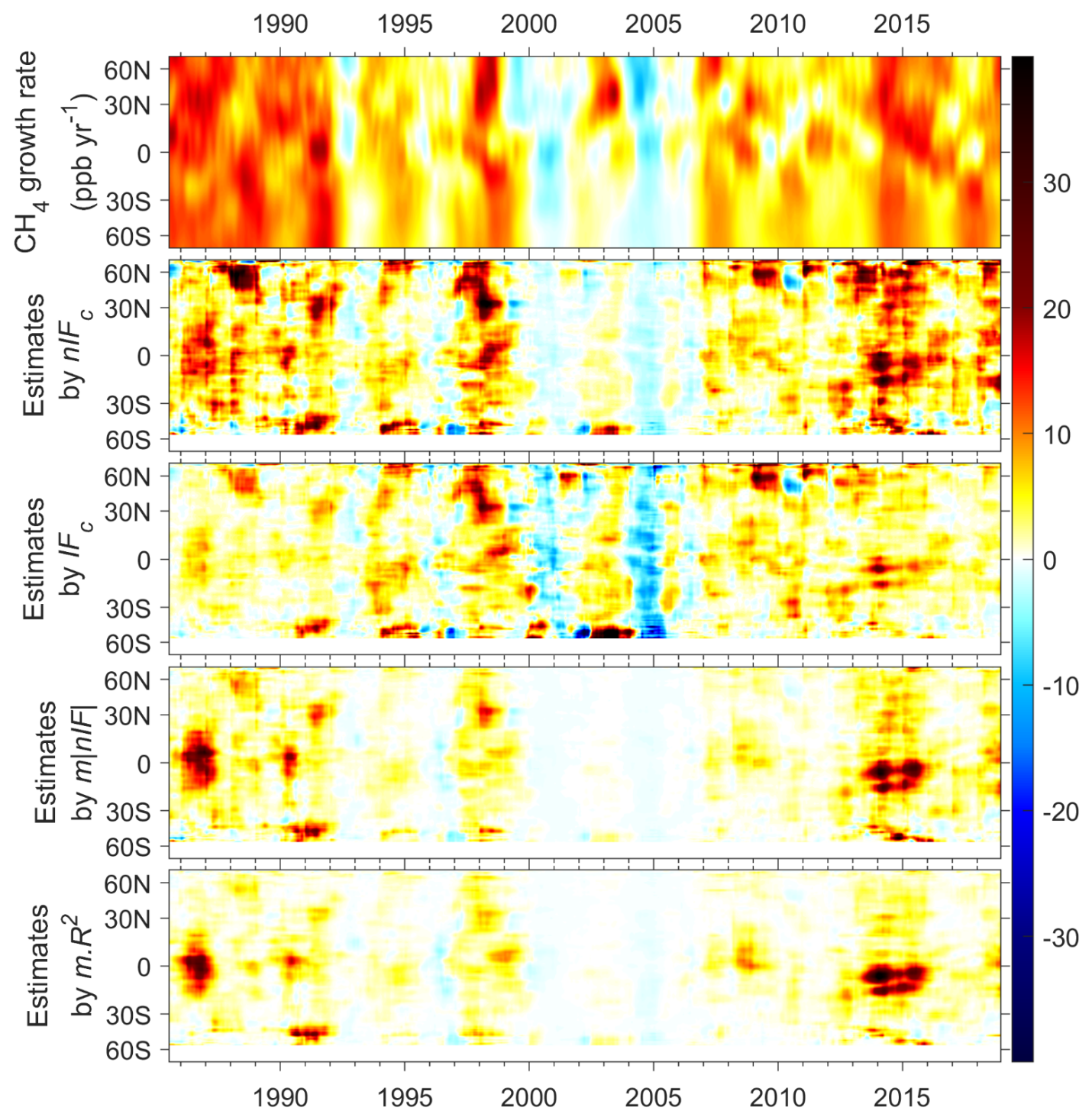

105 Figure 6. Reconstructed zonal $C_{\mathrm{CH} 4}$ varations from observation and estimated climate-driven contributions by various methods based on observational climatic data. The anthropogenic emission and concentration (self-) dependent atmospheric oxidation are considered as "noise" in this analysis. The estimates given by $n I F_{c}$ align the best with observed trends. 
https://doi.org/10.5194/gmd-2021-196

Preprint. Discussion started: 19 July 2021

(c) Author(s) 2021. CC BY 4.0 License.

(c) (i)

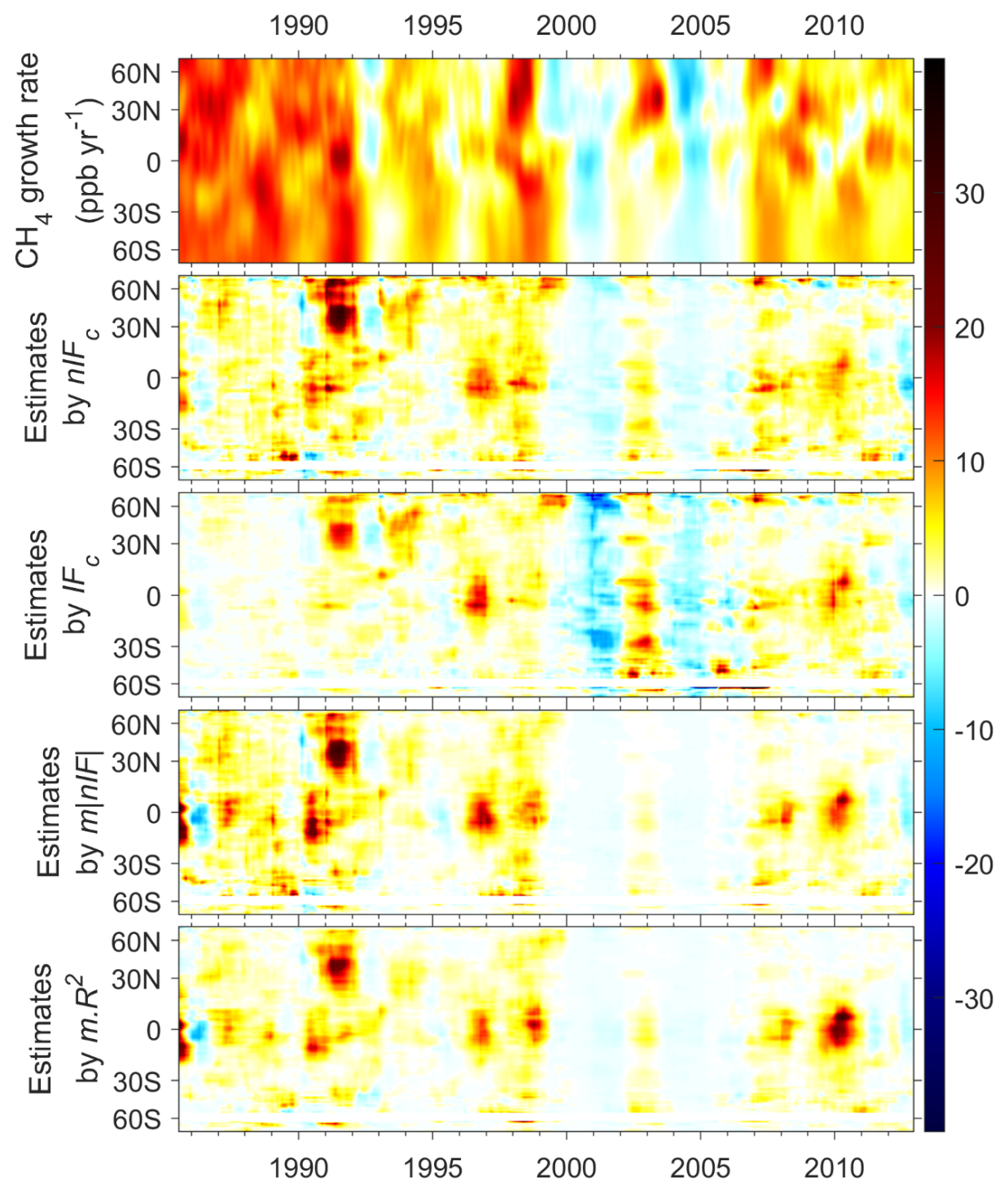

Figure 7. Reconstructed zonal $C_{\mathrm{CH} 4}$ varations from observation and estimated climate-driven contributions by various

110 methods based on simulated climatic data. Unlike Fig. 6, the simulated climatic data fails to reproduce the spatiotemporal pattern of $C_{\mathrm{CH} 4}$ especially at mid-high latitudes. Such gaps indicate the need for model improvement. 
For comparison, Fig. 7 shows the estimated climate-driven contributions to the $C_{\mathrm{CH} 4}$ inferred from the CESM2 simulated climatic data. In contrast to Fig. 6, estimates given by $n I F_{c}$ reflect the observational spatiotemporal pattern of $C_{\mathrm{CH} 4}$ poorly, especially at mid-high latitudes. Figure 7 fails to show the strong signals seen in Fig. 6, such as $0-30^{\circ} \mathrm{S}$ (1988), SH (1991), and NH (1998) instances, and amplifies the 1991 false NH signal. This highlights missing or misrepresented processes of terrestrial $\mathrm{CH}_{4}$ emissions and/or sinks in the CESM2 model. Nevertheless, the estimates by other methods show similar limitations as in Fig. 6. Since other statistical analyses discussed here are less capable in quantifying the causal contributions with observational data, their use for model gap identification and improvement will also be limited.

\section{Discussion and Conclusions}

From our test cases and results above we can make some general points. Firstly, our methodology for the application of the concepts of information flow and normalized information flow is simple and useful. With the potential of quantifying causal contributions between time-series, these methods could help identify missing or misrepresented processes in Earth System Models. We propose a hypothesis to rationalize our findings from the empirical assessments. Finally, we make suggestions for how the method may be further improved.

We have validated four applicable conditions for which we suggest the best approach is to adopt $n I F_{c}$ in the proposed empirical linear equation, as outlined our results (above), while $I F_{c}$ appears to be the best multiplier in those scenarios in which the noisecontribution is large. This simple equation could be widely applied for understanding real-world problems that satisfy at least one of the four conditions. Besides the given example of methane-climate feedbacks, there are a great many problems that would lend themselves to such analysis. We believe that this method is useful for a very large number of feedback systems in Earth systems science as well as other research domains characterized by complexity. Even socio-economic feedbacks could be analyzed, bringing new insight for designing climate-change communications and policies (Cheng et al., in preparation). Nevertheless, the analyzed results from real-world problems have to be interpreted with caution and preferably together with reasonable and consistent physical explanations. For example, we have (earlier) validated the estimated 3D distribution of causal contributions by comparing with the observed 2D and even 3D concentration patterns and built our statistical causal models that are aligned with material balances of $\mathrm{CH}_{4}$ (Cheng and Redfern, under review): Climatic influence on the methane source and sink could behave differently as positive and/or negative feedbacks on interannual to interdecadal timescales, such as via wildfire- $\mathrm{CO}-\mathrm{OH}$ and $S S T-\mathrm{OH}$, possibly leading to a rising $C_{\mathrm{CH} 4}$ even in conditions of negative feedback during a $\mathrm{La}$

140 Niña year (e.g. 2020), in addition to the long-term trend of positive feedback with global warming. This nonlinearity results in complex accelerations and/or decelerations of net methane emissions on various timescales. Since such nonlinearity is the major challenge for process-based Earth system models (Runge et al., 2019) but we show that it can be revealed by quantifying 
causal contributions with $n I F_{c}$. We believe that the methods discussed here will therefore, be valuable for future model assessment and improvement.

As mentioned in section 2.1, the reasonable estimates of causal contributions by $\mathrm{nIF}_{\mathrm{c}}$ suggests that the fractional uncertainty contributed by cause-variable, i.e. $|n I F|$, could be proportional to its physically measured relative causal contribution to the effect-variable, i.e. $\left(\partial Y_{X} / \partial t\right) /(d X / d t)$. Nevertheless, what is the reason behind the inconsistency limitation of $I F_{c}$, and why is such a problem mitigated at high noise levels?

a

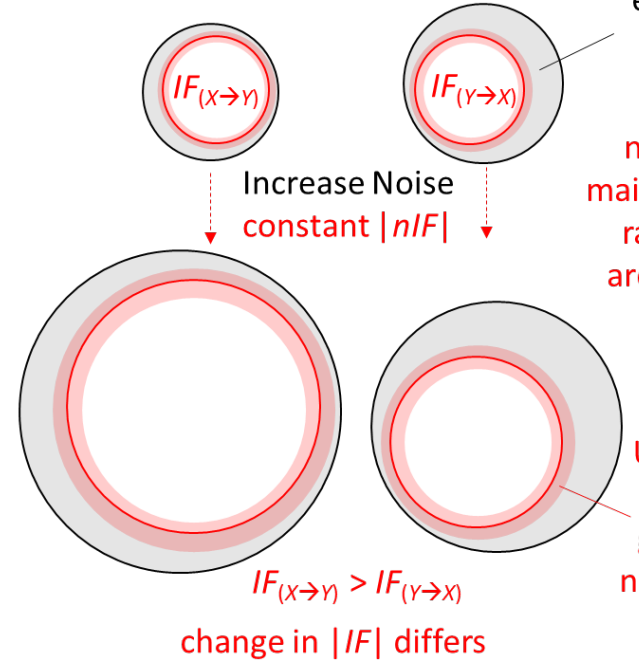

b High Noise Contribution

entropy from Initially, $I F_{(x \rightarrow \eta)}=I F_{(y \rightarrow x)}$

noise

normalization
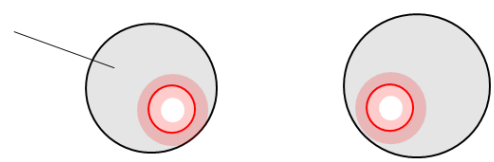

Increase Noise

constant $|n I F|$

ratio between

areas of red and

grey circle

Uncertainty

in entropy

gain due to

normalization

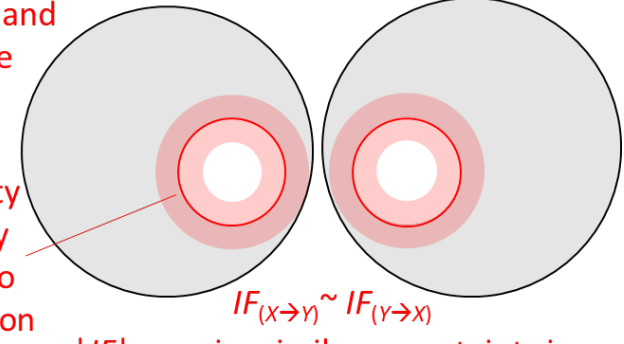

$|I F|$ remains similar, uncertainty in

$|n I F|$ dominates

Figure 8. Illustration of possible reasons behind the observed limitations for $|\boldsymbol{I F}|$ and $|\boldsymbol{n I F}|$ : with low relative noise contributions, the change of noise contribution tends to influence the consistency of $|I F|$; and with high relative noise contributions, the uncertainty due to inherit $|n I F|$ assumption may dominate the error.

Our observations that lie behind this question have been noted in a different context before, where it was seen that the magnitude of noise amplitude may shift the causal direction between two time-series measured by $I F$ (Liang, 2018). Intuitively, although the amount of information (the magnitude of uncertainty or Shannon entropy) is expected to increase with increasing 
amplitude of uncertainty from the noise, the dominating causal direction should remain constant. This dilemma can be rationalized if the dominating causal direction between $X \rightarrow Y$ and $Y \rightarrow X$ is determined by $n I F$ instead of $I F$, in other words, by the relative causal contribution instead of the absolute flow of entropy. Figure $8 \mathrm{a}$ attempts to illustrate a scenario in which there is a relatively weak noise contribution to the total entropy change. A sharp change in noise contribution with a constant $|n I F|$ is represented by an absolute increase of grey area, i.e. increase in marginal entropy $H_{Y}$, with a constant red-circled area fraction. This results in an inconsistent $|I F|$. On the other hand, Fig. $8 \mathrm{~b}$ shows a scenario with strong noise contribution to the total entropy change (large grey area fraction). An increase of noise contribution with an unchanged $|n I F|$ would have a negligible influence on the consistency of $|I F|$; hence the benefit of normalization diminishes but its associated error grows due to the reliance of the noise contribution when estimating $|n I F|$ (equation 4). As seen in equation 7, when the increase in marginal entropy $H_{Y}$ dominates the denominator, $|n I F|$ will be approximately proportional to $|I F|$ and hence also to $\left(\partial Y_{X} / \partial t\right) /(d X / d t)$ as well, while the actual estimate of $|n I F|$ is heavily influenced by the increased marginal entropy.

This method could be further improved. For example, if our hypothesis holds, we can extend it to multi-variable causal contributions (expressed below), subject to the independency between various cause-variables $X_{i}$ :

$\frac{d Y}{d t}=\sum_{i=1}^{n} \alpha_{i}\left(n I F_{c, i}\right) \frac{d X_{i}}{d t}+\frac{\partial Y_{\text {noise }}}{\partial t}=\sum_{i=1}^{n}\left[\left(\theta_{X i \rightarrow Y} \frac{d Y / d t}{d X i / d t}\right) \frac{d X_{i}}{d t}\right]+\frac{\partial Y_{\text {noise }}}{\partial t}$

where $\theta_{X \rightarrow Y}$ represents the fractional contribution to $d Y / d t$ from $d X i / d t$, and the sum of $\theta_{X \rightarrow Y}$ should be less than 1 . However, for real applications, it may be difficult to estimate the calibratiovn factor $\alpha_{\mathrm{i}}$ if the contribution is low; and the interdependencies between $X_{i}$ s may often be assumptions without rigorous justification.

The lead-lag between causes and effects provides another opportunity for further improvement of this methodology. As seen in Fig. 3, the estimates by $n I F_{c}$ also allow a reasonable estimate of the designed time-lead of causes ahead of effects (by comparing sub-Fib $\mathrm{i}, \mathrm{j}$ to $\mathrm{a}, \mathrm{b}$ for periods in green and purple boxes). Using this lead-time estimate, one may revise the selected time-series to better match the series between causes and effects and potentially improve the estimates of correlation sign. Furthermore, the original sign of $I F$ and the estimated error in $I F$ with defined confidence level (e.g. 90\%) (Liang, 2014, 2018) have yet to be used. The estimated error may help to provide a range (instead of just a value) of causal contribution with a defined confidence level. The original sign that indicates increasing or decreasing uncertainty or predictability may also be useful in some ways. Last but not the least, comparison with other causal methods may reveal new insights and possibly improve the applicability of our proposed methodology.

Code and Data Availability: Data sources are all public databases as indicated at the appropriate point in the text. Analysis, represented by the data shown in the Figures, was carried out using standard MatLab routines, with source codes obtainable at dx.doi.org/10.6084/m9.figshare.14985381. 
Author contributions: $\mathrm{CHC}$ conceptualised the project, methodology, data curation and analysis. Both authors contributed to the discussion and manuscript preparation.

Competing interests: The authors declare that they have no conflict of interest.

Acknowledgments: The early phase of this study was supported by the National Key Research and Development Program of China (2017YFA0603804). This work was supported by NTU Singapore SUG to SA TR.

\section{References}

Andor, M. A. and Fels, K. M.: Behavioral Economics and Energy Conservation - A Systematic Review of Non-price Interventions and Their Causal Effects, Ecol. Econ., 148, 178-210, https://doi.org/10.1016/j.ecolecon.2018.01.018, 2018.

Athey, S. and Imbens, G. W.: The State of Applied Econometrics: Causality and Policy Evaluation \%J Journal of Economic Perspectives, 31,3-32, 10.1257/jep.31.2.3, 2017.

205 Bai, C. Z., Zhang, R., Bao, S. L., Liang, X. S., and Guo, W. B.: Forecasting the Tropical Cyclone Genesis over the Northwest Pacific through Identifying the Causal Factors in Cyclone-Climate Interactions, J. Atmos. Ocean. Technol., 35, 247-259, 10.1175/jtech-d-17-0109.1, 2018. Barnett, L., Barrett, A. B., and Seth, A. K.: Solved problems for Granger causality in neuroscience: A response to Stokes and Purdon, NeuroImage, 178, 744-748, https://doi.org/10.1016/j.neuroimage.2018.05.067, 2018.

Chen, A., Gu, Y., Liu, S., DeAngelis, G. C., and Angelaki, D. E.: Evidence for a Causal Contribution of Macaque Vestibular, But Not 210 Intraparietal, Cortex to Heading Perception, 36, 3789-3798, 10.1523/JNEUROSCI.2485-15.2016\%J The Journal of Neuroscience, 2016. Cheng, C.-H. and Redfern, S. A. T.: Impact of Interannual and Multidecadal Trends on Methane-Climate Feedbacks and Sensitivity, Nature Communications, under review.

Cheng, C.-H., Redfern, S. A. T., Lim, J. J., and Chirstopoulos, G.: Social Dynamics from Climate-Justice Movement to Carbon Price Google Trends Analysis, in preparation.

215 Cox, P. M., Huntingford, C., and Williamson, M. S.: Emergent constraint on equilibrium climate sensitivity from global temperature variability, Nature, 553, 319-322, 10.1038/nature25450, 2018.

Danabasoglu, G., Lamarque, J.-F., Bacmeister, J., Bailey, D. A., DuVivier, A. K., Edwards, J., Emmons, L. K., Fasullo, J., Garcia, R., Gettelman, A., Hannay, C., Holland, M. M., Large, W. G., Lauritzen, P. H., Lawrence, D. M., Lenaerts, J. T. M., Lindsay, K., Lipscomb, W. H., Mills, M. J., Neale, R., Oleson, K. W., Otto-Bliesner, B., Phillips, A. S., Sacks, W., Tilmes, S., van Kampenhout, L., Vertenstein,

220 M., Bertini, A., Dennis, J., Deser, C., Fischer, C., Fox-Kemper, B., Kay, J. E., Kinnison, D., Kushner, P. J., Larson, V. E., Long, M. C. Mickelson, S., Moore, J. K., Nienhouse, E., Polvani, L., Rasch, P. J., and Strand, W. G.: The Community Earth System Model Version 2 (CESM2), Journal of Advances in Modeling Earth Systems, 12, e2019MS001916, https://doi.org/10.1029/2019MS001916, 2020.

Friston, K., Parr, T., Zeidman, P., Razi, A., Flandin, G., Daunizeau, J., Hulme, O., Billig, A., Litvak, V., Moran, R., Price, C., and Lambert, C.: Dynamic causal modelling of COVID-19 [version 2; peer review: 2 approved], 5, 10.12688/wellcomeopenres.15881.2, 2020.

225 Granger, C. W. J.: Investigating Causal Relations by Econometric Models and Cross-spectral Methods, Econometrica, 37, 424-438, $10.2307 / 1912791,1969$.

Hall, A., Cox, P., Huntingford, C., and Klein, S.: Progressing emergent constraints on future climate change, Nature Climate Change, 9 , 269-278, 10.1038/s41558-019-0436-6, 2019.

Hill, C. A., Suzuki, S., Polania, R., Moisa, M., O'Doherty, J. P., and Ruff, C. C.: A causal account of the brain network computations 230 underlying strategic social behavior, Nature Neuroscience, 20, 1142-1149, 10.1038/nn.4602, 2017.

Im, S.-H., An, S.-I., Kim, S. T., and Jin, F.-F.: Feedback processes responsible for El Niño-La Niña amplitude asymmetry, Geophysical Research Letters, 42, 5556-5563, https://doi.org/10.1002/2015GL064853, 2015.

Liang, X. S.: Unraveling the cause-effect relation between time series, Physical Review E, 90, 052150, 10.1103/PhysRevE.90.052150, 2014.

Liang, X. S.: Normalizing the causality between time series, Physical Review E, 92, 10.1103/PhysRevE.92.022126, 2015. 
235 Liang, X. S.: Information flow and causality as rigorous notions ab initio, Physical Review E, 94, 052201, 10.1103/PhysRevE.94.052201, 2016 .

Liang, X. S.: Causation and information flow with respect to relative entropy, Chaos, 28, 10.1063/1.5010253, 2018.

Lin, S.-H. and Ikram, M. A.: On the relationship of machine learning with causal inference, European Journal of Epidemiology, 35, 183$185,10.1007 / \mathrm{s} 10654-019-00564-9,2020$.

240 Luo, Y., Peng, J., and Ma, J.: When causal inference meets deep learning, Nature Machine Intelligence, 2, 426-427, 10.1038/s42256-0200218-x, 2020.

Nowack, P., Runge, J., Eyring, V., and Haigh, J. D.: Causal networks for climate model evaluation and constrained projections, Nature Communications, 11, 1415, 10.1038/s41467-020-15195-y, 2020.

Ornes, S.: Core Concept: How does climate change influence extreme weather? Impact attribution research seeks answers, Proceedings of

245 the National Academy of Sciences, 115, 8232-8235, 10.1073/pnas.1811393115, 2018.

Pearl, J.: The seven tools of causal inference, with reflections on machine learning, 62, 54-60, 10.1145/3241036, 2019.

Pfrommer, T., Goeschl, T., Proelss, A., Carrier, M., Lenhard, J., Martin, H., Niemeier, U., and Schmidt, H.: Establishing causation in climate litigation: admissibility and reliability, Climatic Change, 152, 67-84, 10.1007/s10584-018-2362-4, 2019.

Rasmussen, S. A., Jamieson, D. J., Honein, M. A., and Petersen, L. R.: Zika Virus and Birth Defects - Reviewing the Evidence for Causality,

250 374, 1981-1987, 10.1056/NEJMsr1604338, 2016.

Runge, J., Bathiany, S., Bollt, E., Camps-Valls, G., Coumou, D., Deyle, E., Glymour, C., Kretschmer, M., Mahecha, M. D., Muñoz-Marí,

J., van Nes, E. H., Peters, J., Quax, R., Reichstein, M., Scheffer, M., Schölkopf, B., Spirtes, P., Sugihara, G., Sun, J., Zhang, K., and Zscheischler, J.: Inferring causation from time series in Earth system sciences, Nature Communications, 10, 2553, 10.1038/s41467-01910105-3, 2019.

255 Russo, F. and Williamson, J.: Epistemic Causality and Evidence-Based Medicine, History and Philosophy of the Life Sciences, 33, 563-581, 2011.

Schreiber, T.: Measuring Information Transfer, Physical Review Letters, 85, 461-464, 10.1103/PhysRevLett.85.461, 2000.

Seth, A. K., Barrett, A. B., and Barnett, L.: Granger Causality Analysis in Neuroscience and Neuroimaging, 35, 3293-3297, 10.1523/JNEUROSCI.4399-14.2015\%J The Journal of Neuroscience, 2015.

260 Stips, A., Macias, D., Coughlan, C., Garcia-Gorriz, E., and San Liang, X.: On the causal structure between CO2 and global temperature, Scientific Reports, 6, 21691, 10.1038/srep21691, 2016.

Stokes, P. A. and Purdon, P. L.: A study of problems encountered in Granger causality analysis from a neuroscience perspective, 114, E7063E7072, 10.1073/pnas.1704663114\%J Proceedings of the National Academy of Sciences, 2017.

Sugihara, G., May, R., Ye, H., Hsieh, C.-h., Deyle, E., Fogarty, M., and Munch, S.: Detecting Causality in Complex Ecosystems, 338, 496-

$265500,10.1126 /$ science. $1227079 \%$ J Science, 2012.

Swain, D. L., Singh, D., Touma, D., and Diffenbaugh, N. S.: Attributing Extreme Events to Climate Change: A New Frontier in a Warming World, One Earth, 2, 522-527, https://doi.org/10.1016/j.oneear.2020.05.011, 2020.

Varian, H. R.: Causal inference in economics and marketing, 113, 7310-7315, 10.1073/pnas.1510479113 \%J Proceedings of the National Academy of Sciences, 2016.

270 Vázquez-Patiño, A., Campozano, L., Mendoza, D., and Samaniego, E.: A causal flow approach for the evaluation of global climate models, 40, 4497-4517, https://doi.org/10.1002/joc.6470, 2020.

Verbitsky, M. Y., Mann, M. E., Steinman, B. A., and Volobuev, D. M.: Detecting causality signal in instrumental measurements and climate model simulations: global warming case study, Geosci. Model Dev., 12, 4053-4060, 10.5194/gmd-12-4053-2019, 2019.

Weber, T., Wiseman, N. A., and Kock, A.: Global ocean methane emissions dominated by shallow coastal waters, Nature Communications,

275 10, 4584, 10.1038/s41467-019-12541-7, 2019.

Wieder, W., Butterfield, Z., Lindsay, K., Lombardozzi, D., and Keppel-Aleks, G.: Seasonal drivers of carbon cycle interannual variability 2 represented by the Community Earth System Model (CESM2), Global Biogeochemical Cycles, submitted.

Winkler, A. J., Myneni, R. B., Hannart, A., Sitch, S., Haverd, V., Lombardozzi, D., Arora, V. K., Pongratz, J., Nabel, J. E. M. S., Goll, D.

S., Kato, E., Tian, H., Arneth, A., Friedlingstein, P., Jain, A. K., Zaehle, S., and Brovkin, V.: Slow-down of the greening trend in natural

280 vegetation with further rise in atmospheric CO2, Biogeosciences Discuss., 2021, 1-36, 10.5194/bg-2021-37, 2021. 